\title{
Wie der politische Diskurs auch läuft, am Ende entscheidet die Mehrheit, oder nicht?
}

\section{Kommentar zum Hauptbeitrag von Ingo Pies}

[1] In seinem interessanten Plädoyer präsentiert Ingo Pies seine Ordonomik als Methode, um ein Versagen des öffentlichen Diskurses, das er diagnostiziert und neben Markt- und Politikversagen stellt, zu beheben oder zu vermeiden. Er zielt damit auf eine aktuell auffällige Entwicklung, nämlich die (’populistischen`) Polarisierungstendenzen, die dazu führen, dass sich die Antagonismen zwischen den politischen Positionen verhärten. Die Gesellschaft spaltet sich in unversöhnliche Lager, die sich im Vorfeld demokratischer Wahlen zunehmend wegen ihrer politischen Positionen anfeinden und jeweils diskriminiert und unterworfen fühlen, wenn das Wahlergebnis zu ihren Ungunsten ausfällt. Beispiele solcher Zuspitzungen gibt es in westlichen Demokratien derzeit genügend: die Pro- und KontraTrump-Agitation im jüngsten US-Wahlkampf, die Konfrontation zwischen Anhängern und Gegner der Nationalen Front vor den französischen Präsidentenwahlen und, besonders ausgeprägt, die Auseinandersetzungen vor und nach der Volksabstimmung über den Brexit in Großbritannien. Pies scheut sich nicht, seinen ordonomischen Ansatz einer konkreten Bewährungsprobe auszusetzen und wählt dafür die Konfrontation in der aktuellen öffentlichen Debatte über die >richtige< Flüchtlingspolitik in Deutschland.

[2] Diese Courage ist anerkennenswert und auch die Denkanstöße, die Pies in seinem Aufsatz gibt, sind wertvoll - wenngleich vielleicht eher für die Klärung des Problems als für eine Lösung, auf die er hinarbeitet. In meinem Kommentar möchte ich mich auf drei Einwände konzentrieren, die aus meiner Sicht die vorgeschlagene Rolle der Ordonomik in Frage stellen. Die Einwände beziehen sich im Wesentlichen auf die Funktion des öffentlichen Diskurses und einer möglichen ordonomischen Mediation desselben. M.E. darf dabei nicht ignoriert werden, dass dieser Diskurs periodisch in Mehrheitsentscheide durch demokratische Abstimmung mündet.

[3] Der erste Einwand gilt der grundlegenden Analogie zu Hobbes Reflektionen über die Anarchie und ihre Überwindung in seinem Leviathan. Wird diese Analogie den Bedingungen des öffentlichen Diskurses in Demokratien wirklich gerecht werden? Pies übersetzt Hobbes Beschreibung des Dilemmas, das der Kampf aller gegen alle mit sich bringt, in ein Gefangenendilemma. In diesem würden sich alle besser stehen, wenn sie den Kampf einstellten. Dies ist eine geläufige Interpretation. An sie knüpft eine Vielzahl theoretischer Vorschläge an, wie das

* Prof. Dr. Ulrich Witt, Max-Planck-Institut für Menschheitsgeschichte (vormals für Ökonomik), Kahlaische Str.10, D-07745 Jena, E-Mail: witt@evoecon.mpg.de. 
Dilemma überwunden werden kann. (Pies setzt dabei auf eine Überführung des Gefangendilemmas in ein Koordinationsspiel, wie er sie in Hobbes Argumentation angelegt sieht (vgl. [34]ff.).) Zunächst ist jedoch zu fragen, ob das Gefangenendilemma tatsächlich die Situation abbildet, wie sie sich bezüglich der Meinungsbildung in der Öffentlichkeit darstellt.

[4] In demokratischen Gesellschaften dient der öffentliche Diskurs zum einen dem Ringen um die Formulierung von Entscheidungsalternativen für die regelmäBig stattfindenden Abstimmungen im Zuge politischer Wahlen. Zum anderen dient der Diskurs dem Werben um Stimmen für die jeweiligen Alternativen im Vorfeld dieser Wahlen. Ich meine, dass das Gefangenendilemma diese Struktur nicht erfasst, jedenfalls nicht, solange von der Abstimmung nicht ein einstimmiges Ergebnis verlangt wird. Nehmen wir zur Veranschaulichung das Gefangendilemma in der Abbildung 2 im Text von Pies. Der angestrebten Analogie zu Hobbes entsprechend wären im ersten Schritt in der Abbildung die beiden Strategien >Individuelle Waffenruhe - ja/nein< zu ersetzen durch Strategien die >Bereitschaft im gesellschaftlichen Diskurs aufeinander zuzugehen - ja/nein< genannt werden sollen. Der Logik des Gefangenendilemmas zufolge wäre wechselseitiges Aufeinander-Zugehen dann für alle das Beste.

[5] Das ist jedoch keineswegs evident - anders als bei Hobbes, wo die Vermeidung endloser anarchischer Kämpfe als Pareto-Optimum einleuchtet. Der Gewinn eines wechselseitigen Aufeinander-zugehens soll den Parteien ja auch erst durch Konstruktion von >Überbietungsargumenten $<$ - ein zentraler Teil der Ordonomik demonstriert werden. Damit stehen den widerstreitenden Parteien jedoch nicht unmittelbar verfügbare Optionen bereit (wie im Falle von Hobbes Ruhenlassen der Waffen). Vielmehr hängen ihre Optionen von erst noch herzustellenden institutionellen Kompromiss-Regelungen ab. Im Beispiel der Flüchtlingskontroverse sind die institutionellen Regelungen sogar auf internationale Unterstützung angewiesen. Da winkt also ein Kooperationsgewinn bei einem Aufeinander-Zugehen nur, wenn darüber hinaus diverse weitere Konditionalitäten erfüllt sind. (Was das bedeutet, wird in meinem zweiten Einwand unten näher beleuchtet.)

[6] Der Natur des Gefangenendilemmas entsprechend würde sich die kooperative Lösung des wechselseitigen Aufeinander-Zugehens (also Zelle I in Abb. 2) auch dann nicht spontan einstellen, wenn sie für die widerstreitenden Lager das Beste wäre. (Die Lösung ist kein Gleichgewichtspunkt in dem Spiel.) Damit soll das Diskursversagen modelltheoretisch beschrieben werden. Um es zu beheben, wird der zweite Schritt vollzogen, den Pies aus seiner Analogie zu Hobbes ableitet. Er besteht in der Überführung des Gefangenendilemmas in ein Koordinationsspiel. Dafür müssen die Auszahlungen in den Zellen II und IV der Spielmatrix den Wert 0 annehmen. Wie das zustande kommt, habe ich nicht ganz verstanden. Solange man sich an die Logik des Bimatrix-Spiels hält, kann man die Änderung der Auszahlungen gegenüber dem Gefangenendilemma jedenfalls nicht mit einer Abmachung außerhalb des Spiels begründen. Diese Abmachung muss selbst als Teil der Strategiewahl aus einem Spiel hervorgehen.

[7] Darüber hinaus lassen sich auch interpretatorische Zweifel anmelden. Das wechselseitige Drohpotential, durch das Hobbes eine stets gleiche Strategiewahl 
als erzwingbar ansieht, folgt aus der Möglichkeit, die verlustreichen Kämpfe endlos fortzusetzen. Dagegen ist das in Analogie zu Hobbes konstruierte Drohpotential der widerstreitenden Diskursparteien (das die Überführung in das Koordinationsspiel sichern soll) doch sehr viel bescheidener: die Drohung damit, nicht aufeinander zuzugehen, stellt ja nur in Aussicht, über die Kontroverse bei den nächsten Wahlen oder dem nächsten Volksentscheid per demokratischer Abstimmung entscheiden zu lassen. Schließlich besteht der Zweck von politischen Wahlen und Volksentscheiden ja gerade darin, über kontroverse Positionen in der öffentlichen Meinung nach der Mehrheitsregel zu entscheiden, weil allseits Konsens findende Kompromisse in großen Gemeinwesen utopisch sind. Im Ergebnis würde sich durch den Wahlentscheid eines der widerstreitenden Lager durchsetzen. Die jeweils unterlegene Position bliebe einflusslos, in der Wirkung also so, als ob sie sich vollständig auf die Position der Wahlgewinner zubewegt hätte. Das heißt, bei einem wechselseitigen Beharren auf der Strategie des Nicht-aufeinander-Zugehens würde der Wahlausgang ein Ergebnis schaffen, das sich nicht von dem in Zelle II oder Zelle IV des Gefangenendilemmas unterscheidet.

[8] Der Gedanke, dass sich die Parteien durch ein strategisches, wechselseitiges Aufeinander-Eingehen im öffentlichen Diskurs einen Gefallen tun können, ist an sich einleuchtend. Aber die Begründung dafür führt m.E. nicht zum Modell des Gefangenendilemmas. Gerade weil dem Wettstreit im öffentlichen Diskurs durch periodische Mehrheitsabstimmung Entscheidungen über die widerstreitenden Positionen folgen, sollten sich die an der Meinungsbildung teilnehmenden Parteien strategisch verhalten. Denn der Ausgang von Wahlen und Volksentscheiden ist ja stets ungewiss und man sollte sich überlegen, wie man im Falle einer Niederlage dasteht. Angenommen, die widerstreitenden Parteien würden rein rational entscheiden, dann müssten sie - gegebenenfalls durch mehr oder weniger provokative Modifikation der eigenen Festlegungen - versuchen, die Position der Gegenseite über den öffentlichen Diskurs so zu manipulieren, dass der eigene Schaden bei einer Wahlniederlage in angemessenem Rahmen bleibt bzw. bei Risikoaversion minimiert wird. Ein Aufeinander-Zugehen wird man das nicht nennen können, und die Wechselseitigkeit des Manipulationsinteresses sieht auch eher nach einem Null-Summenspiel als nach einem Positiv-Summenspiel aus.

[9] Mein zweiter Einwand bezieht sich auf die Rolle, die Überbietungsargumente spielen können, um die widerstreitenden Parteien im öffentlichen Diskurs aus der polarisierenden Lagerbildung herauszuführen. Wenn, wie eben erwähnt, der Versuch unternommen wird, die Position der Gegenseite zu manipulieren, um damit vielleicht den größten Schaden bei eigener, späterer Wahlniederlage abzumildern, dann erfolgt das aus eigenem Antrieb. Der Ordonomik zufolge soll es dagegen einen Mediator des öffentlichen Diskurses geben. Dieser soll, vermittelt durch >Überbietungsargumente`, nach politischen Handlungsoptionen suchen, die die moralischen Positionen der widerstreitenden Lager noch besser erfüllen, zugleich aber einen Kompromiss zwischen ihnen darstellen.

[10] Mir ist an dieser Konstruktion zum einen nicht klar geworden, wer die unparteiische Mediator-Rolle spielen soll und kann - Journalisten? Sozialwissenschaftler wie Pies selbst? Zum anderen werden, wie schon erwähnt, den wider- 
streitenden Parteien nur erst noch herzustellende institutionelle KompromissRegelungen in Aussicht gestellt, falls sie sich auf die Mediation einlassen. Doch wer garantiert deren Herstellung? Was geschieht, wenn es zu Realisierungsschwierigkeiten und Abweichungen kommt? Ist nicht zu erwarten, dass rational entscheidende Teilnehmer am öffentlichen Diskurs solche Unwägbarkeiten bei ihrer Zustimmungs- bzw. Ablehnungsentscheidung einbeziehen? Sollte dies der Fall sein, tritt neben die ethischen Fragen eine verhaltensökonomische Frage, nämlich die nach der Risikopräferenz der involvierten Parteien. Je größer die Risikoaversion, umso mehr dürften die Chancen für eine Zustimmung zum Kompromiss schwinden. Statt des Aufeinander-zugehens käme es dann wohl doch zum sshow down in der Wahlnacht.

[11] Mit dem Hinweis auf die Rationalität der widerstreitenden Parteien im öffentlichen Diskurs ziehe ich allerdings selbst eine mögliche verhaltensökonomische Kritik auf mich. Bei emotional besetzten Themen, wie dem der Flüchtlingsproblematik, dürfte rationales Verhalten im öffentlichen Diskurs eher nicht zu erwarten sein. Das ist auch ein Problem für die ordonomischen Vorschläge von Pies, das mich zu meinem dritten Einwand veranlasst. Die Warte der konsequentialistischen Ethik, von der aus er argumentiert, setzt zum einen ein hohes Maß an Rationalität bei den widerstreitenden Lagern voraus. Wenn an die Stelle rationaler Beurteilung von Konsequenzen aber eine emotionale Pro- oder Kontra-Haltung tritt, dann treffen auch gut gemeinte Vermittlungsvorschläge auf taube Ohren. Ist nicht gerade die eingangs erwähnte Polarisierungstendenz, die dazu führt, dass sich die Antagonismen zwischen den politischen Positionen verhärten, eine Folge des Appells an Emotionen? Ist dieser Appell nicht entweder selbst emotional begründet oder machiavellistisch motiviert - also in beiden Fällen ethischen Argumenten schwer oder gar nicht zugänglich?

[12] Zum anderen setzt die konsequentialistische >Diskursethik auf die Überzeugungskraft von Argumenten, die auf die moralischen Konsequenzen der Entscheidungen verweisen. Ein solcher Appell versagt jedoch bekanntermaßen, wenn sich wesentliche Teilnehmer des Diskurses an moralischen Urteilen mit Absolutheitsanspruch orientieren und eben nicht an einer konsequentialistischen Ethik. Es scheint, dass das gerade im Zusammenhang mit der Flüchtlingsproblematik häufig der Fall ist. Dann werden die Ohren einem Abwägen moralisch besserer oder schlechterer Alternativen wie im Aufsatz von Pies (z.B. Abwägen zwischen Hilfe für mobile junge Flüchtlinge hier und jetzt vs. Hilfe für all die schwachen auf dem Fluchtweg oder gar daheim hängen gebliebenen) ebenfalls verschlossen bleiben. Emotionale Blockade ebenso wie moralischer Fundamentalismus sind gute Gründe, sich in Demokratien nicht darauf zu verlassen, dass der öffentliche Diskurs zu tragfähigen Kompromissen oder gar Konsens führt. Periodische demokratische Mehrheitsentscheidungen beseitigen nicht notwendig ein Diskursversagen. Aber sie schaffen eine Klarheit, die selbst ein funktionierender Diskurs nicht schaffen kann.

[13] Die formulierten Einwände sind für Pies möglicherweise nicht neu. Er wird für den einen oder anderen vielleicht schon die Gegenargumente parat haben. Ich 
bin auf diese gespannt - im Sinne einer Fortsetzung eines anregenden Diskurses, den er mit seinem Aufsatz eröffnet hat.

\section{Neoliberale Ideologie}

Folgen und Alternativen

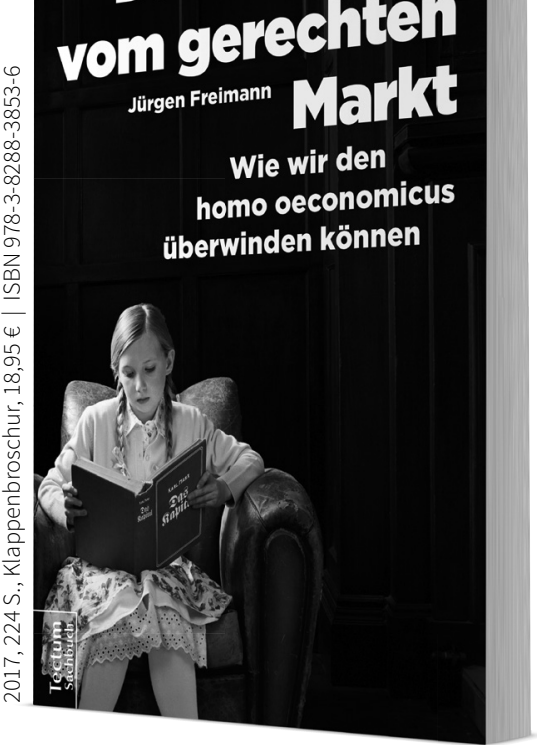

Bis zum Herbst 2016 waren CETA, TTIP und Co in aller Munde. Tausende Menschen protestierten gegen die sogenannten Freihandelsabkommen, aber die politisch verantwortlichen hielten beharrlich an ihnen fest.

Was können wir selbst dazu beitragen, dem gefräßigen Markt Grenzen zu setzen?

Welche Auswirkungen hat die neoliberale Ideologie für Menschen, Gesellschaften und Staaten? 